\title{
Application of Land Use Change and Prediction in Urban Planning Evaluation and Formulation
}

\author{
Huipeng Liao, Mixuan Liu, Huagui He, Weijun Yang, Liang Guo, Yang Liu, Keyun Chen, \\ Zhaoxia Chen \\ ${ }^{1}$ Guangzhou Urban Planning \& Design Survey Research Institute, Guangzhou, China \\ ${ }^{2}$ Guangdong Enterprise Key Laboratory for Urban Sensing, Monitoring and Early Warning, Guangzhou, China \\ Email: *liaohuipeng163@163.com
}

How to cite this paper: Liao, H.P., Liu, M.X., He, H.G., Yang, W.J., Guo, L., Liu, Y., Chen, K.Y. and Chen, Z.X. (2021) Application of Land Use Change and Prediction in Urban Planning Evaluation and Formulation. Journal of Computer and Communications, 9, 230-237.

https://doi.org/10.4236/jcc.2021.96015

Received: June 8, 2021

Accepted: June 27, 2021

Published: June 30, 2021

\begin{abstract}
The study monitored land use change in Nansha during 2000-2018, evaluate Nansha's urban planning by the overlay analysis of land use general planning, land use distribution, and economic data, and predicted the urban expansion in 2025 and 2035 to provide scientific information for urban planning in the future. The results showed that the built-up area rapidly expanded $\left(78.32 \mathrm{~km}^{2}\right.$ to $153.75 \mathrm{~km}^{2}$ in $2000-2018$ ), and the GDP and the newly-added enterprises remarkably increased as well. Most of built-up area matched the land use planning. The urban expansion was simulated to $168.27 \mathrm{~km}^{2}$ in 2025 and to $207.04 \mathrm{~km}^{2}$ in 2035 for future land use planning.
\end{abstract}

\section{Keywords}

Land Use/Land Cover Change, Urban Expansion Simulation, Urban Planning

\section{Introduction}

Remote sensing (RS) is a fast, convenient, and economical way to monitor land use in long-time series. Supervised classification, such as minimum distance classification and maximum likelihood classification, and unsupervised classification, such as IsoData and K-Means, are two main approaches to extract vegetation, water, built-up area, bare land from RS data.

Land use classification provides information for investigating land resources and formulating spatial planning, and thus is essential to policy-making and usage control of land use [1]. Understanding the relationship between land use and urban planning, combined with analyses of economic development, can evaluate urban planning implementation and provide guidance to land use polices. 
In addition, Land use change can be predicted to assist urban planning by quantitative simulation models and spatial simulation models. The quantitative simulation model, such as Markov chain (MC) model, can estimate and predict the trend and quantity of land use change [2]. The spatial simulation model, such as Future Land-Use Simulation (FLUS) model [3], the optimized model of Cellular Automata model, is able to simulate land use spatial distribution. FLUS model can examine the complex relationship between various land use types and economic, social and environmental factors. It is suitable for simulating complex and longterm land use changes and has the advantages of convenient operation and higher accuracy compared to other models [3] [4] [5].

The purpose of the study was to use RS-derived land use change data and economic data to evaluate urban planning implementation, and simulate urban expansion to provide reliable information and scientific basis for the adjustment of regional development policies and planning.

\section{Method}

\subsection{Study Area and Data Source}

Nansha, located at $22^{\circ} 26^{\prime}-23^{\circ} 06^{\prime}$ north and $113^{\circ} 13^{\prime}-113^{\circ} 43^{\prime}$ east, with the total area of $783.86 \mathrm{~km}^{2}$, is a district of south Guangzhou, China. In the last two decades, Nansha rapidly developed from a countryside to the subcenter of the city. The study selected Nansha as the study area to explore how the land use changed during the development, and how the land use can be applied to urban planning evaluation and formulation.

The spatial datasets of the study include Landsat images in 2000-2018, urban land use general planning (ULUGP) for 2006-2020 and industrial parks' boundary, as well as enterprises' location, which were obtained from United States Geological Survey (https://www.usgs.gov/), Guangzhou government, and Baidu Map API, respectively. The statistical datasets were Nansha's Gross Domestic Product (GDP) in 2005-2018, basic information of enterprises in 2018, and revenue data of 2017, which were obtained from Nansha's statistical yearbook (available from 2005), National Enterprise Credit Information Publicity System, and Guangzhou government, respectively.

\subsection{Land Use Change Detection in Nansha}

Landsat images were preprocessed by radiometric calibration, atmospheric correction, geometric correction, and mosaic. Supervised classification was applied to detect vegetation, water, built-up area, and bare land during 2000-2018 to analyze urban development of the study area. Urban expansion speed and expansion intensity were calculated based on [6]. For urban expansion speed, $>10 \mathrm{~km}^{2} /$ year was defined as high-speed expansion, $6-10 \mathrm{~km}^{2} /$ year was rapid expansion, 2 - 6 $\mathrm{km}^{2} /$ year was medium-speed expansion, and $<2 \mathrm{~km}^{2} /$ year was low-speed expansion. For urban expansion intensity, $>1.92 \%$ was defined as high-speed expansion, $1.05 \%-1.92 \%$ was rapid expansion, $0.59 \%-1.05 \%$ was medium-speed ex- 
pansion, $0.28 \%-0.59 \%$ was low-speed expansion, and $<0.28 \%$ was slow expansion.

\subsection{Application of Land Use in Urban Planning Evaluation}

Urban planning of Nansha was evaluated in two perspectives. One was to understand how the urban development matched the plan by the overlay analysis of land use distribution and urban land use planning. The other perspective was to examine how the economic development was related to land use change and affected by urban planning through the statistical analysis of GDP growth and enterprise growth, the hot spot analysis of enterprise distribution (adjusted by revenue) by Kernel density model [7], and the analysis of the spatial relationship between industrial parks and enterprises.

\subsection{Urban Expansion Simulation in Nansha, 2025 and 2035}

The study applied MC model and FLUS model to predict the land demand and spatial distribution of built-up area of Nansha in 2025 and 2035, with the basis on the land use distribution in 2018, urban planning data (with restrictions such as prime farmland protection area, range of ecological redline, etc.), DEM, slope, aspect, distance to roads, and distance to administrative centers. All the factors were converted into units of $30 \mathrm{~m} \times 30 \mathrm{~m}$. The theory of MC model and FLUS model are as follows:

1) MC Model

MC model was based on the progression of the formation of Markov stochastic process systems for the prediction of one status being changed to another [8]. The future land use changes conditional probability formulas are:

$$
\begin{gathered}
S(t+1)=P_{i j} \times S(t) \\
P_{i j}=\left(\begin{array}{cccc}
P_{11} & P_{12} & \cdots & P_{1 n} \\
P_{21} & P_{22} & \cdots & P_{2 n} \\
\vdots & \vdots & & \vdots \\
P_{n 1} & P_{n 2} & \cdots & P_{n n}
\end{array}\right)
\end{gathered}
$$

and $\left(0 \leq P_{i j}<1\right.$ and $\left.\sum_{j=1}^{N} P_{i j}=1,(i, j=1,2, \ldots, n)\right)$

Where $S(t)$ is the state of the system at time $t, S(t+1)$ is the state of the system at time $(t+1) ; P_{i j}$ is the matrix of transition probability in a state.

(2) FLUS Model

FLUS model was applied to simulate spatial distribution of land use. In the model, the overall conversion probability $T P_{p, k}^{t}$ of the land use type $k$ of the grid $p$ at time $t$ is obtained by considering the suitability probability of the grid, the adaptive inertia coefficient, and the neighborhood effect and the conversion cost [3]:

$$
T P_{p, k}^{t}=P_{p, k} \times \Omega_{p, k}^{t} \times \text { Ineria }_{k}^{t} \times\left(1-s C_{c \rightarrow k}\right)
$$

where $P_{p, k}, \Omega_{p, k}^{t}$ and Ineria $t_{k}^{t}$ are suitability probability, neighborhood effect, adaptive inertia coefficient, respectively; $s c_{c \rightarrow k}$ is the conversion cost from land 
use type $c$ to land use type $k$.

After estimating the overall probability of each iteration, the FLUS model uses a roulette selection mechanism to determine the land use of each pixel [5].

\section{Results}

\subsection{Land Use Change in Nansha, 2000-2018}

The result showed that the built-up area expanded from $78.32 \mathrm{~km}^{2}$ to $153.75 \mathrm{~km}^{2}$ in 2000-2018, which almost doubled during the 18 years (Figure 1). The annual average of urban expansion speed was $4.19 \mathrm{~km}^{2}$ per year, classified as mediumspeed expansion. The urban expansion intensity was $0.53 \%$ per year, classified as low-speed expansion. The proportion of built-up area grew from $10.7 \%$ to $21.0 \%$ while vegetation decreased from $63.0 \%$ to $53.3 \%$. The proportion of water and bare land was relatively stable in the range of $25.0 \%-26.0 \%$ and $0.2 \%-2.5 \%$, respectively.

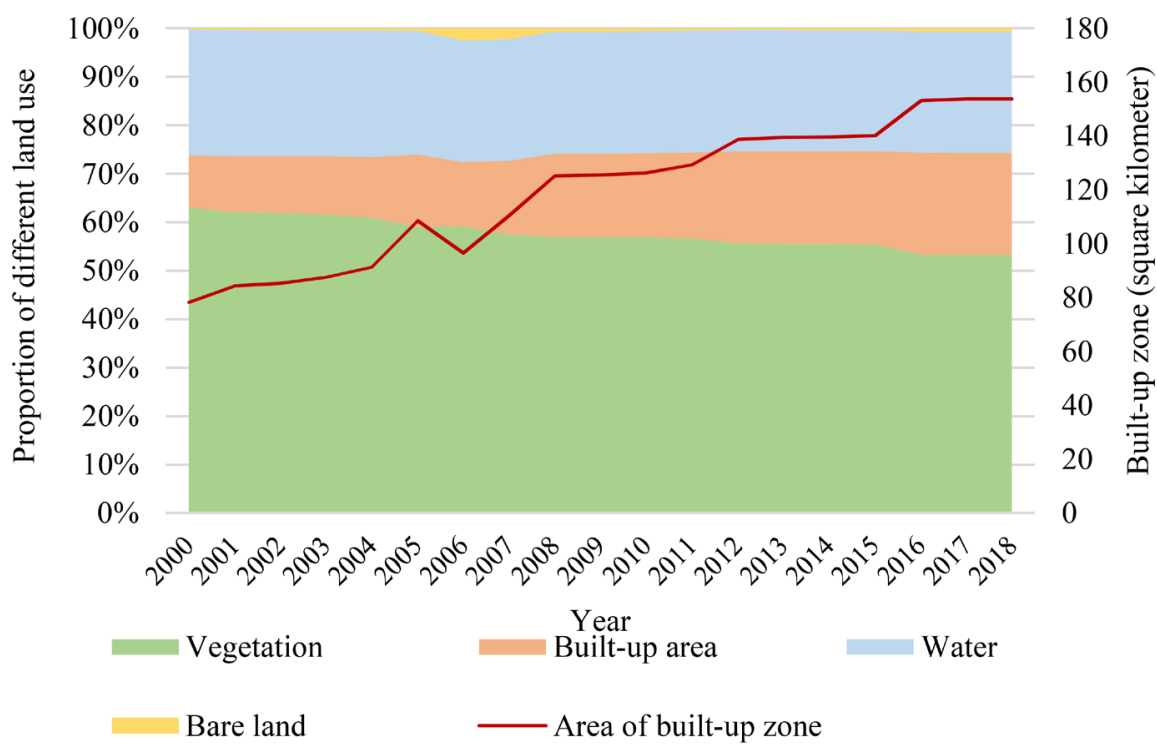

Figure 1. Land use change of Nansha, 2000-2018.

\subsection{Evaluation of Urban Planning in Nansha by Land Use and Economic Development}

In general, the land use distribution in 2018 followed the ULUGP. As Figure 2 showed, in Jiaomen River, Nansha Bay and Chang'an Industrial Park, most of the planned built-up area were developed. Nansha Harbor was developed ahead of the plan as some areas were not planned but built, meaning that its land demand was higher than expectation. The development progress was slow in Sansha village, Hengli Town and Xinlian village, Zhujiang street and Wanqingsha town since most of planned built-up area remain undeveloped by 2018 .

As shown from Figure 3, Nansha's GDP rapidly increased since 2005, especially in 2012. The GDP of the secondary industry was dominant during the period, but the growth slowed after 2014, while the GDP of the tertiary sector 


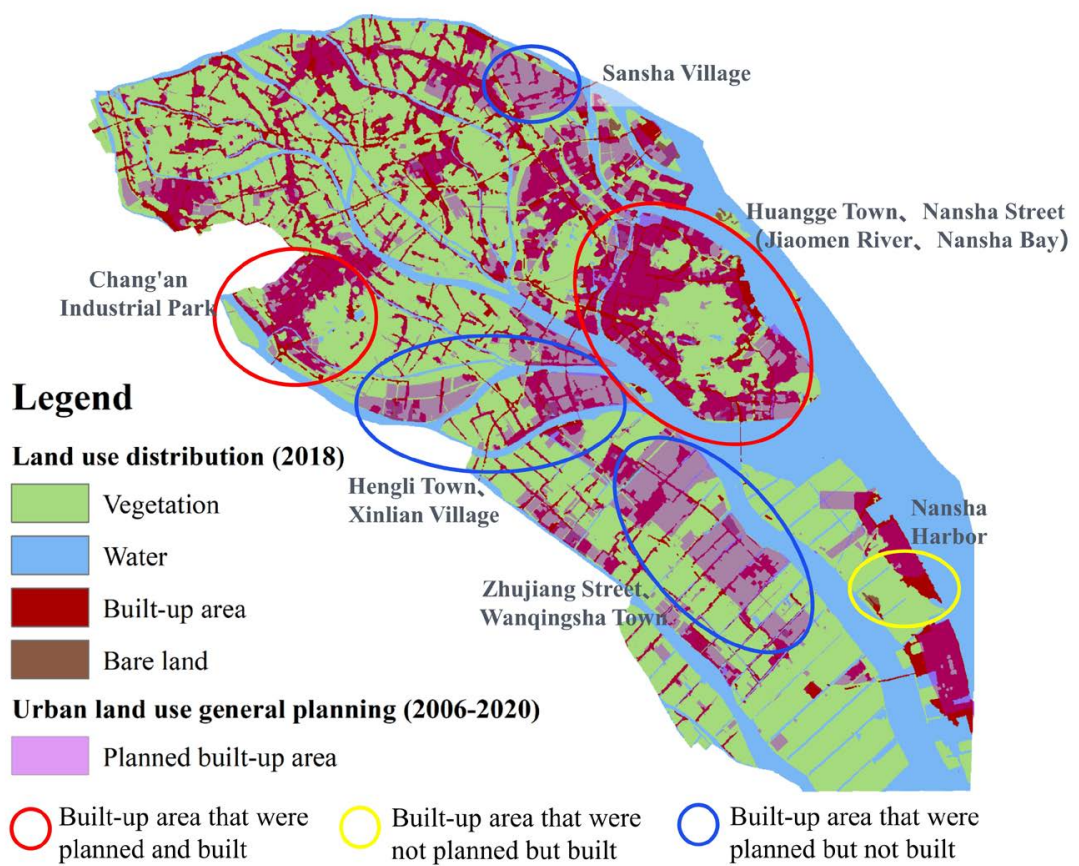

Figure 2. Overlay analysis of ULUGP and land use distribution in 2018.

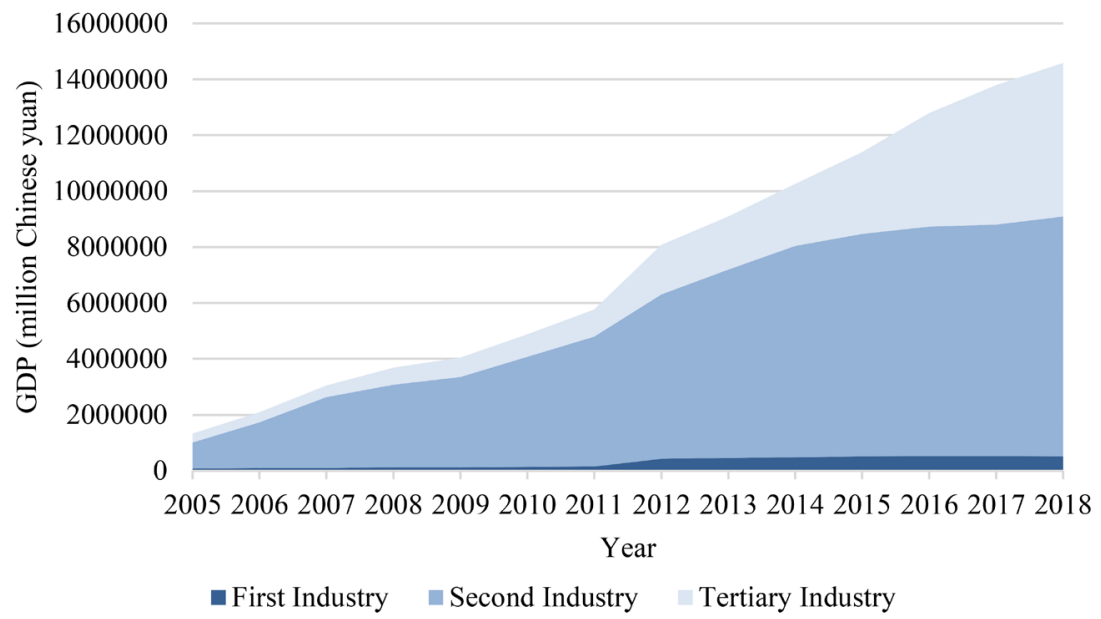

Figure 3. Nansha's GDP in 2005-2018.

remarkably increased. Similarly, the number of newly-added industrial enterprises increased during 2000-2018 in general (Figure 4). After a short decrease between 2011 and 2012, newly-added industrial enterprises increased remarkably after 2012, reflecting the attraction of Nansha in economy.

Figure 5(a) indicated that the enterprises were concentrated in the central and northern area, especially the Jiaomen River, Nansha Bay, and Chang'an Industrial Park. A hot spot fell in Guangzhou Nansha economic and technological development zone, while the aggregation in other industrial parks were not significant. After the adjustment of revenue, hot spots were mainly located in Huangge Town, where had automobile manufacturing companies with high tax payment, such as GAC Toyota (Figure 5(b)). 


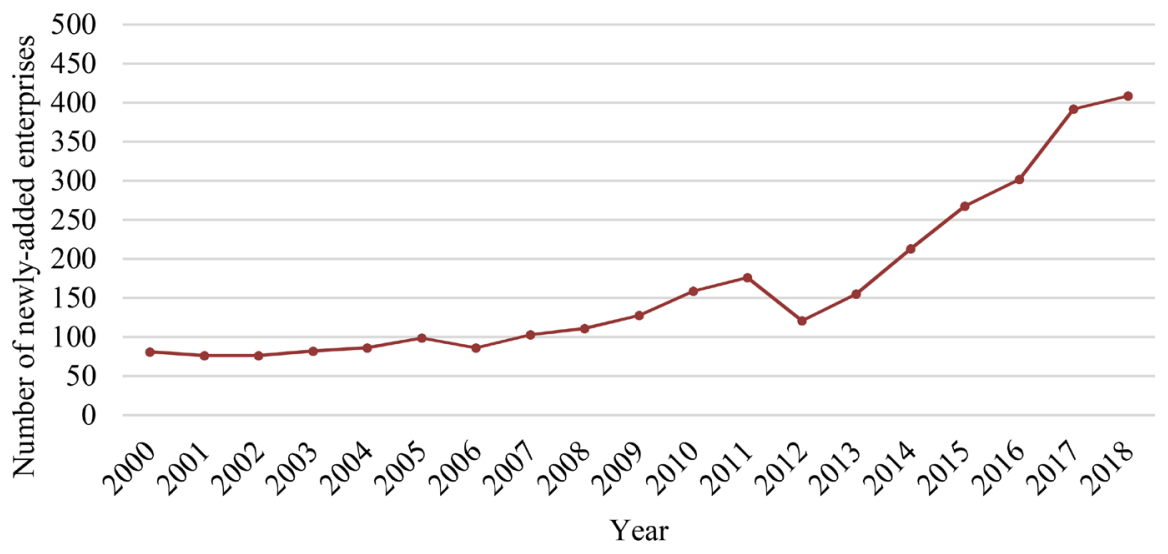

Figure 4. Number of newly-added industrial enterprises in Nansha (2000-2018).

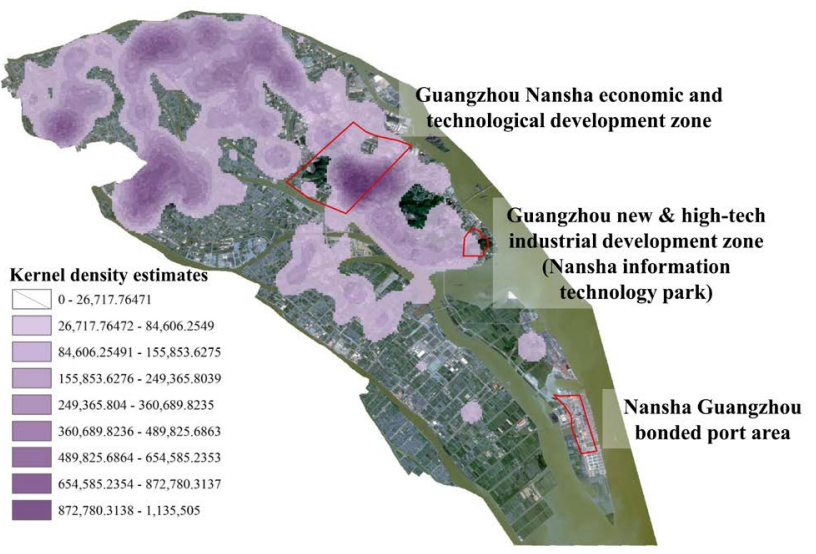

(a)

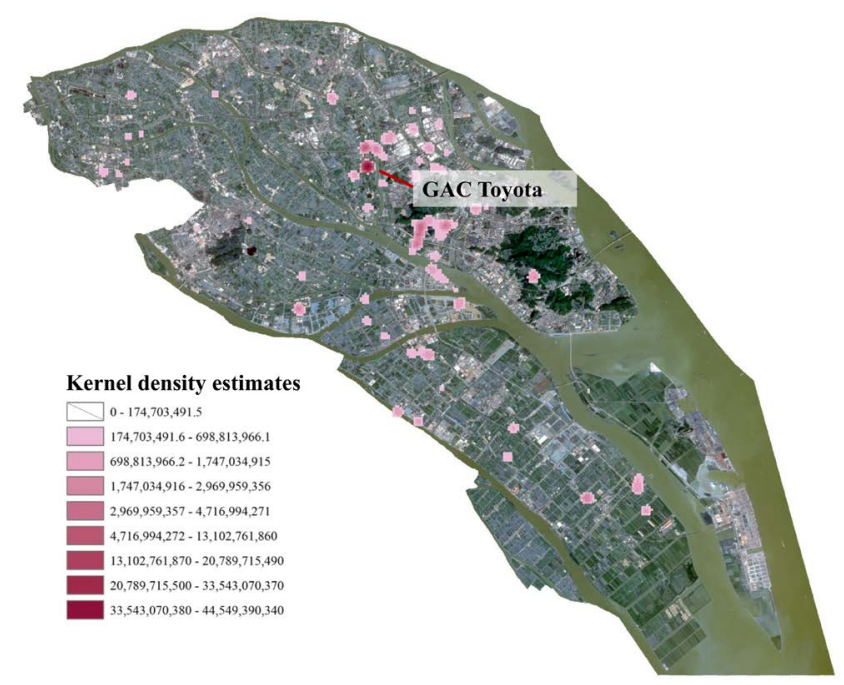

(b)

Figure 5. Hot spot analysis of industrial enterprises in Nansha. ((a): Hot spot analysis of number of industrial enterprises; (b): Hot spot analysis of number of industrial enterprises (revenue-adjusted)).

\subsection{Urban Expansion Simulation in Nansha, 2025 and 2035}

The predicted built-up area of Nansha in 2025 and 2035 will be $168.27 \mathrm{~km}^{2}$ and $207.04 \mathrm{~km}^{2}$, respectively, with an increase of $14.51 \mathrm{~km}^{2}$ and $53.28 \mathrm{~km}^{2}$ respectively compared to 2018 (Figure 6). From 2018 to 2035, the predicted average

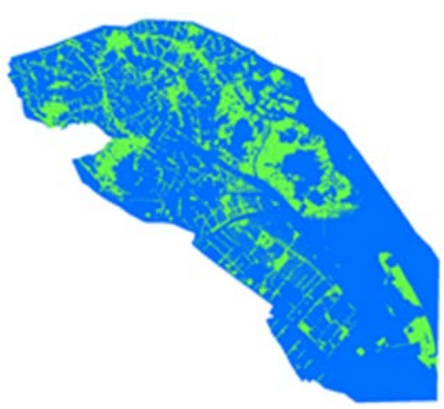

(a)

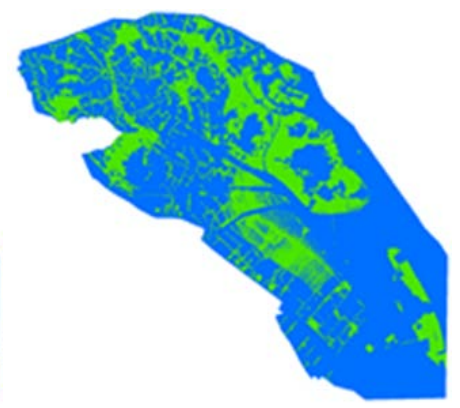

(b)

Figure 6. Urban expansion simulation map of Nansha. (a) Year 2025; (b) Year 2035. 
annual urban expansion speed was $3.13 \mathrm{~km}^{2}$ (medium-sized expansion), and the urban expansion intensity was $0.43 \%$ (low-speed expansion).

\section{Discussion}

The study monitored land use change in Nansha during 2000-2018, evaluate Nansha's urban planning by the overlay analysis of land use general planning, land use distribution, and economic data. The study also predicted the urban expansion in 2025 and 2035, providing scientific information for urban planning and policy-making in the future.

As the results shown, Nansha experienced doubled urban expansion, rapid GDP growth, and remarkable increase of enterprises, indicating the booming economy of the area during 2000-2018. However, some places did not match the expectation of urban planning. Nansha Harbor had high land demand as its builtup area exceeded the planned area, while Sansha village, Hengli Town and Xinlian village, Zhujiang street and Wanqingsha town developed slower than planned with much unbuilt area. The study suggests further research on the unmatched area to understand the underlying reasons for developing ahead or behind, which may assist adjustment of urban planning in the future.

Since the study monitored about two decades of land use change in Nansha, Landsat is the most appropriate satellite data for long time-series analysis. However, as its spatial resolution is only $30 \mathrm{~m}$, Landsat-derived land use classification is less precise than the classification derived from other satellites with high resolution, such as Gaofen-1 (launched in 2013) and Gaofen-2 (launched in 2014) satellites. As time goes by, future research my consider using Gaofen-1 and Gaofen-2 satellites instead to monitor land use change in decades.

\section{Conclusion}

The study used RS-derived land use data and economic data to evaluate urban planning implementation, and predict urban expansion for urban planning in Nansha. The results indicated a rapid growth of built-up area, GDP, and number of enterprises during 2000-2018. Most places developed as planned. The urban expansion simulation predicted a medium-sized and low-speed expansion in 2035. Future research may further explore the underlying reasons for developing ahead of or behind planning and enlarge study areas with using high resolution satellite data.

\section{Acknowledgements}

The authors thanks to the support of Key-Area Research and Development Program of Guangdong Province (2020B0101130009), and the Guangdong Enterprise Key Laboratory for Urban Sensing, Monitoring and Early Warning (2020B121202019).

\section{Conflicts of Interest}

The authors declare no conflicts of interest regarding the publication of this paper. 


\section{References}

[1] Cao, X., Ouyang, S. and Lyu, C. (2021) Detailed Compilation of National Land Spatial Planning Based on Land Use Categorization. Economic Geography, 41, 192-200

[2] Al-sharif, A.A. and Pradhan, B. (2014) Monitoring and Predicting Land Use Change in Tripoli Metropolitan City Using an Integrated Markov Chain and Cellular Automata Models in GIS. Arabian journal of geosciences, 7, 4291-4301. https://doi.org/10.1007/s12517-013-1119-7

[3] Liu, X., Liang, X., Li, X., Xu, X., Ou, J., Chen, Y. and Pei, F. (2017) A Future Land Use Simulation Model (FLUS) for Simulating Multiple Land Use Scenarios by Coupling Human and Natural Effects. Landscape and Urban Planning, 168, 94-116. https://doi.org/10.1016/j.landurbplan.2017.09.019

[4] Huang, K., Dai, W.Y., Huang, W.L. and Ou, H. (2020) Study on the Delimitation of Urban Growth Boundary Based on FLUS Model and Kinetic Energy Theorem. Journal of Geo-information Science, 22, 557-567.

[5] Wang, C.B., Li, A.N., Zhang, X.R., Nan, X. and Bian, J.H. (2021) Multi-Scenario Simulation of Land Use Change along China-Pakistan Economic Corridor through Coupling FLUS Model with SD Model. Journal of Geo-information Science, 36, 6578.

[6] Zhan, Q., Dong, R. and Xiao, Y. (2018) The Spatial-Temporal Evolution and Comparative Study of Urban Land Cover of Asian and African Cities. https://doi.org/10.12677/ULU.2018.61001

[7] Silverman, B.W. (1986) Density Estimation for Statistics and Data Analysis. Chapman and Hall, New York, p. 76. https://doi.org/10.1007/978-1-4899-3324-9

[8] Muller, M.R. and Middleton, J. (1994) A Markov Model of Land-Use Change Dynamics in the Niagara Region, Ontario, Canada. Landscape Ecology, 9, 151-157. 